\title{
Clinician Attitudes Regarding ICD Deactivation in DNR/DNI Patients
}

\author{
Andrew J. Bradley, MD*, Adam D. Marks, MD, MPH² \\ ${ }^{1}$ Division of Cardiology, George Washington University, Washington, D.C.; '2Department of Internal Medicine, University of Michigan, Ann Arbor, \\ Michigan.
}

BACKGROUND: Implantable cardioverter-defibrillators (ICDs) offer lifesaving therapies but can become burdensome at the end of life. Many ICD patients choose to implement a do-not-resuscitate/do-not-intubate (DNR/DNI) order. When hospitalized, patients are seen by a range of clinicians whose beliefs about ICD management in DNR/DNI patients may vary.

OBJECTIVE: To assess clinician opinions on managing ICDs in DNR/DNI patients and stratify it by specialty and training level.

METHODS: An online survey was sent to attending physicians, fellows, advanced practice providers (physician assistants and nurse practitioners), and residents in general internal medicine, cardiology, electrophysiology, and geriatrics at an academic medical center. Residents were compared to attending physicians, and attending physicians were additionally stratified by specialty.

RESULTS: The response rate was 32\%, yielding $161 \mathrm{com}-$ plete responses. Among residents $(n=73), 49.3 \%$ were com- fortable with discussing ICD deactivation and $16.4 \%$ asked about it routinely. By contrast, among attending physicians ( $\mathrm{n}$ $=66$ ), $78.8 \%$ were comfortable with discussing deactivation and $34.8 \%$ routinely asked. Fewer general internists $(19.2 \%$ of inpatient internists, $10.5 \%$ of outpatient internists) routinely asked about ICD deactivation as compared with $83.3 \%$ of geriatricians and $73.3 \%$ of cardiologists/electrophysiologists. Twenty-one percent of all respondents felt a DNR/DNI order equated to requesting ICD deactivation; Heart Rhythm Society (HRS) guidelines favor a more nuanced approach.

CONCLUSIONS: Residents are less comfortable discussing ICD deactivation than attending physicians and do so less frequently. General internists discuss deactivation less routinely than cardiologists and geriatricians. Many providers hold opinions about ICD deactivation that differ from HRS guidelines. Additional didactic education could help close these gaps in clinician practice. Journal of Hospital Medicine 2017;12:498-502. (c) 2017 Society of Hospital Medicine
Implantable cardioverter-defibrillators (ICDs) offer lifesaving therapies to many patients and have been implanted in hundreds of thousands of patients. ${ }^{1}$ The population of patients with ICDs is growing rapidly, and the national ICD Registry reports over 12,000 devices are implanted monthly. ${ }^{2}$ This population includes patients with congenital heart disease, ischemic cardiomyopathy, and idiopathic arrhythmias. If these patients experience ventricular tachycardia or fibrillation, an ICD attempts to restore sinus rhythm and prevent death. While a shock from an ICD may be lifesaving, it can be a traumatic and startling experience for the patient and perhaps distressful for families to witness. ${ }^{3,4}$

Although ICDs are intended to save lives, they do not slow the progress of the patient's underlying cardiac and noncardiac comorbidities. All these patients will eventually die, whether from their cardiac disease or another condition. The literature includes many anecdotes about patients shocked multiple times by their defibrillator while actively dying. ${ }^{4}$ These situations could be prevented with preemptive ICD deactivation. (ICDs can function not only as car-

\footnotetext{
*Address for correspondence and reprint requests: Andrew J. Bradley, Division of Cardiology, George Washington University, 2150 Pennsylvania Avenue NW, Washington, D.C. 20037; Telephone: 202-741-2323; Fax: 202-741-2324; E-mail: ajbrad@email.gwu.edu
}

Received: July 20, 2016; Revised: November 30, 2016; Accepted: December 13, 2016

2017 Society of Hospital Medicine DOI 10.12788/jhm.2762 dioverters and defibrillators, as implied by their name, but also as pacemakers. "Deactivation" as used in this paper refers only to disabling the tachycardia therapies. No distinction was made between defibrillation with a shock and anti-tachycardia pacing.) Therefore, research on ICD deactivation has emphasized patients who are acutely terminally ill, while less emphasis has been placed on patients who are not actively dying. ${ }^{4-8}$

Patients may, for a variety of reasons, request a do-not-resuscitate/do-not-intubate (DNR/DNI) order as their code status. However, it is not necessarily clear what a DNR/DNI order implies for ICD management. One survey of attending physicians found that $19 \%$ of respondents felt a DNR/DNI order was equivalent to requesting ICD deactivation. ${ }^{9}$ On the other hand, patients are split on whether they would want their device deactivated while in hospice or even at the very end of life. ${ }^{6}$ Heart Rhythm Society (HRS) guidelines favor a nuanced approach to ICD deactivation in DNR/DNI patients that emphasizes the individual patient's comorbidities and goals. ${ }^{10} \mathrm{~A}$ patient's individual circumstances might justify a choice to be DNR/DNI without deactivating the ICD. Decision-making in these circumstances requires a careful conversation between the patient and clinician. It is important to identify barriers that might prevent optimal shared decision-making.

Clinicians have been surveyed on ICD management at the end of life, but these studies have generally focused on attending physicians. ${ }^{5,9,11}$ However, physician trainees (ie, 
TABLE. Demographic Data of Survey Respondents ${ }^{a}$

\begin{tabular}{|c|c|c|c|c|c|c|c|}
\hline & \multicolumn{7}{|c|}{ Clinical role } \\
\hline & PGY1 & PGY2 & $\mathrm{PGY3/4}$ & Fellow & PA/NP & Attending & Total \\
\hline \multicolumn{8}{|l|}{ Specialty } \\
\hline Internal Medicine Resident & 24 & 21 & 28 & -- & -- & -- & 73 \\
\hline General Medicine, Outpatient & -- & -- & -- & -- & 1 & 26 & 27 \\
\hline General Medicine, Inpatient & -- & -- & -- & -- & -- & 19 & 19 \\
\hline Cardiology & -- & -- & -- & 8 & 4 & 13 & 25 \\
\hline Electrophysiology & -- & -- & -- & 1 & 2 & 2 & 5 \\
\hline Geriatrics & -- & -- & -- & -- & 6 & 6 & 12 \\
\hline Total & 24 & 21 & 28 & 9 & 13 & 66 & 161 \\
\hline
\end{tabular}

residents and fellows) as well as advanced practice providers (ie, physician assistants and nurse practitioners) are responsible for much of the clinical care provided to hospitalized patients. In particular, they are often the clinicians to discuss code status with patients. Different specialties (eg, car-

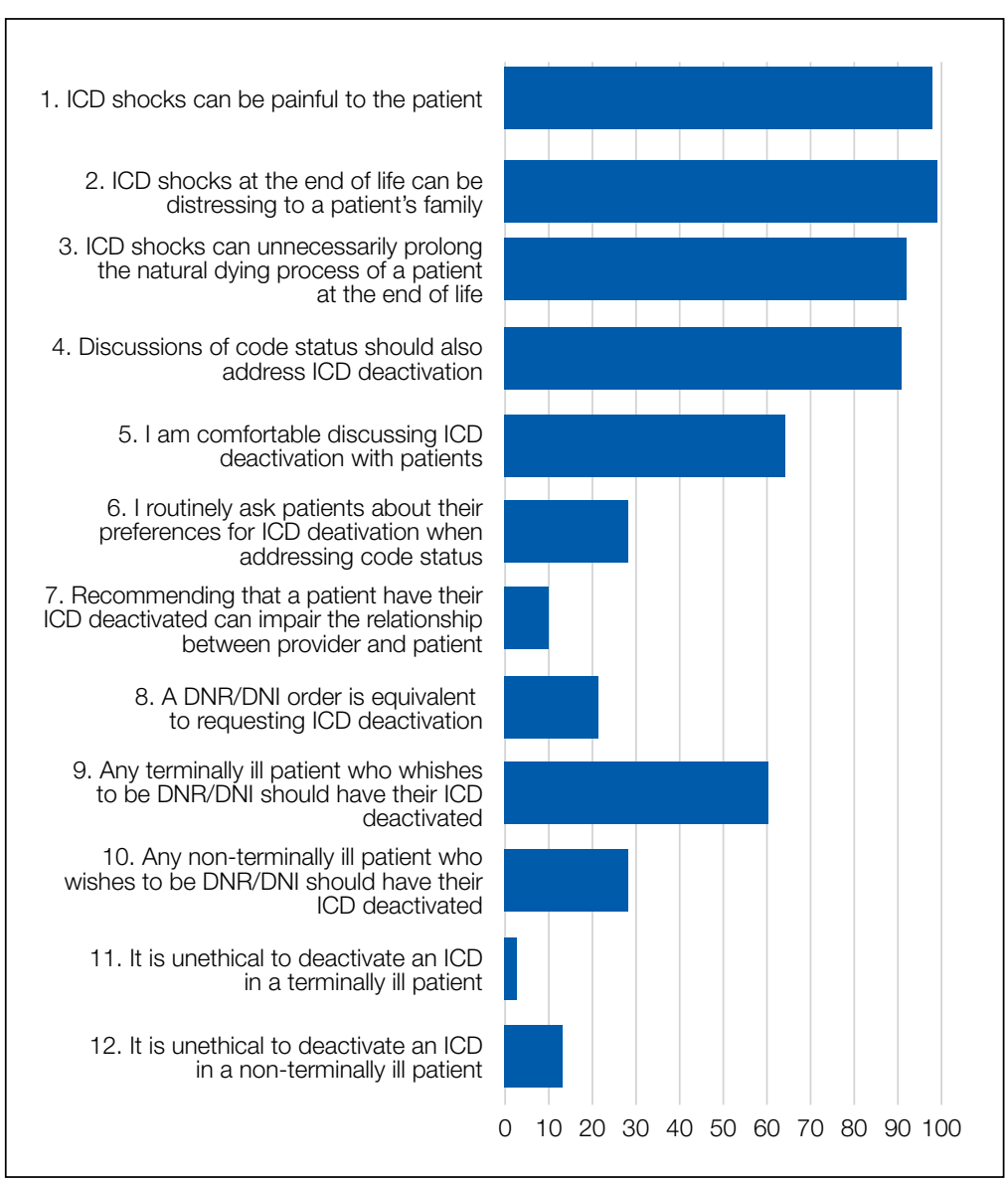

FIG. 1. Answers of all respondents. $X$-axis indicates the percentage giving an affirmative answer, defined as either "agree" or "strongly agree." diology, general medicine, and geriatrics) manage different sets of patients, which might affect clinicians' opinions on ICD management. We therefore designed a survey to assess clinician attitudes and beliefs regarding ICD deactivation, including in non-terminally ill patients, and to evaluate for differences according to training level and specialty.

\section{METHODS}

Case-based and Likert-scale questions were considered for this survey, with the latter being chosen for ease of completion by respondents. An online survey tool (SurveyMonkey; San Mateo, CA) was used for data collection; no identifying data were collected. E-mail invitations to participate were sent to a combination of mailing lists and individual addresses for residents, fellows, advanced practice providers, and attending physicians in general internal medicine, cardiology, electrophysiology, and geriatrics. The survey remained open for 2.5 weeks. It was conducted 5 months into the academic year, thus trainees were well-established in their current roles. Two $\$ 25$ gift cards were offered to respondents who entered their e-mail into a drawing; responses were not tied to e-mail addresses. Approval for the study was obtained from the University of Michigan Institutional Review Board.

The survey posed 12 questions assessing general attitudes about ICDs as well as individual beliefs and behaviors relating to ICD deactivation. Answers were on a Likert scale of 1 to 5 with 1 representing "strongly disagree" and 5 representing "strongly agree." A score of 3 indicated "unsure or neutral." The first 3 questions appeared together on the first page and were prefaced with "Please 
respond to the following statements about ICD shocks." The next 9 were likewise grouped on the next page and were prefaced with "Please respond to the following statements about ICD deactivation." All 12 questions are shown in Figures 1 and 2. Respondents could easily return to previous questions and change their answers. The survey ended with a third page showing 3 multiple choice demographic questions. The demographic questions were about clinical role (first-, second-, third-, or fourth-year resident, fellow, advanced practice provider, and attending), specialty, and number of ICD deactivations the respondent had been directly involved in $(0,1$ to 5,5 to 10 , and more than 10$)$. Specialty options were internal medicine resident, inpatient general medicine, outpatient general medicine, cardiology, electrophysiology, and geriatrics.

Likert scale answers of "agree" or "strongly agree" were grouped together as an affirmative response, while all other answers were grouped together as a nonaffirmative response. For analysis, residents were grouped together and their responses compared with attending physicians as a group. Additional analysis was done comparing attending physicians stratified by clinical specialty. Given the small number of responses from attending electrophysiologists, they were grouped with attending cardiologists for analysis. Due to the limited number of fellows and advanced practice providers who responded, further evaluation of these groups was not performed. Finally, the number of ICD deactivations respondents had been involved in was stratified by training level. All comparisons were performed using the two-tailed Pearson's chisquared test.

\section{RESULTS}

A total of 170 responses were collected from 508 individuals on the e-mail lists. Two responses were from registered nurses who were not part of the intended study sample and 7 responses were incomplete, having only answered the first 3 questions. These 9 responses were excluded from further analysis, yielding an overall response rate of $32 \%$. The demographics of the remaining 161 respondents are shown in Table 1. Figure 1 shows overall responses to each question.

When comparing residents to attending physicians, there were no statistically significant between-differences except on questions 5 and 6. Specifically, residents were less comfortable than attending physicians discussing ICD deactivation and did so with less regularity $(P<.001$ and $P$ $=.018$, respectively; Figure 2). Comfort levels improved markedly with experience: $29.2 \%$ of interns expressed comfort asking about ICD deactivation as compared with $60.7 \%$ of thirdand-fourth year residents and $78.8 \%$ of attending physicians. Furthermore, comfort level seemed to differences. parallel the regularity with which respondents asked about ICD deactivation: $4.2 \%$ of interns routinely asked about ICD deactivation as compared with $21.4 \%$ of third- and fourth-year residents and $34.8 \%$ of attending physicians.

The only statistically significant difference when comparing attending physicians by specialty was on question 6 of the survey with the groups being unequal in their reliability at asking about ICD deactivation during code status discussions $(P<.001$; Figure 2$)$. Of cardiologists and electrophysiologists, $73.3 \%$ said they routinely ask about ICD deactivation, as well as $83.3 \%$ of geriatricians. By contrast, only $19.2 \%$ of outpatient general internists and $10.5 \%$ of inpatient general internists (ie, hospitalists) said they routinely ask about ICD deactivation.

There were no differences between groups when asked whether ICD deactivation was part of a DNR/DNI order (question 8), or if an ICD should be deactivated in DNR/ DNI patients (questions 9 and 10). As shown in Figure 1, $21.1 \%$ of respondents felt that a DNR/DNI order is equivalent to requesting ICD deactivation, $60.2 \%$ felt that terminally ill DNR/DNI patients should have their device de-

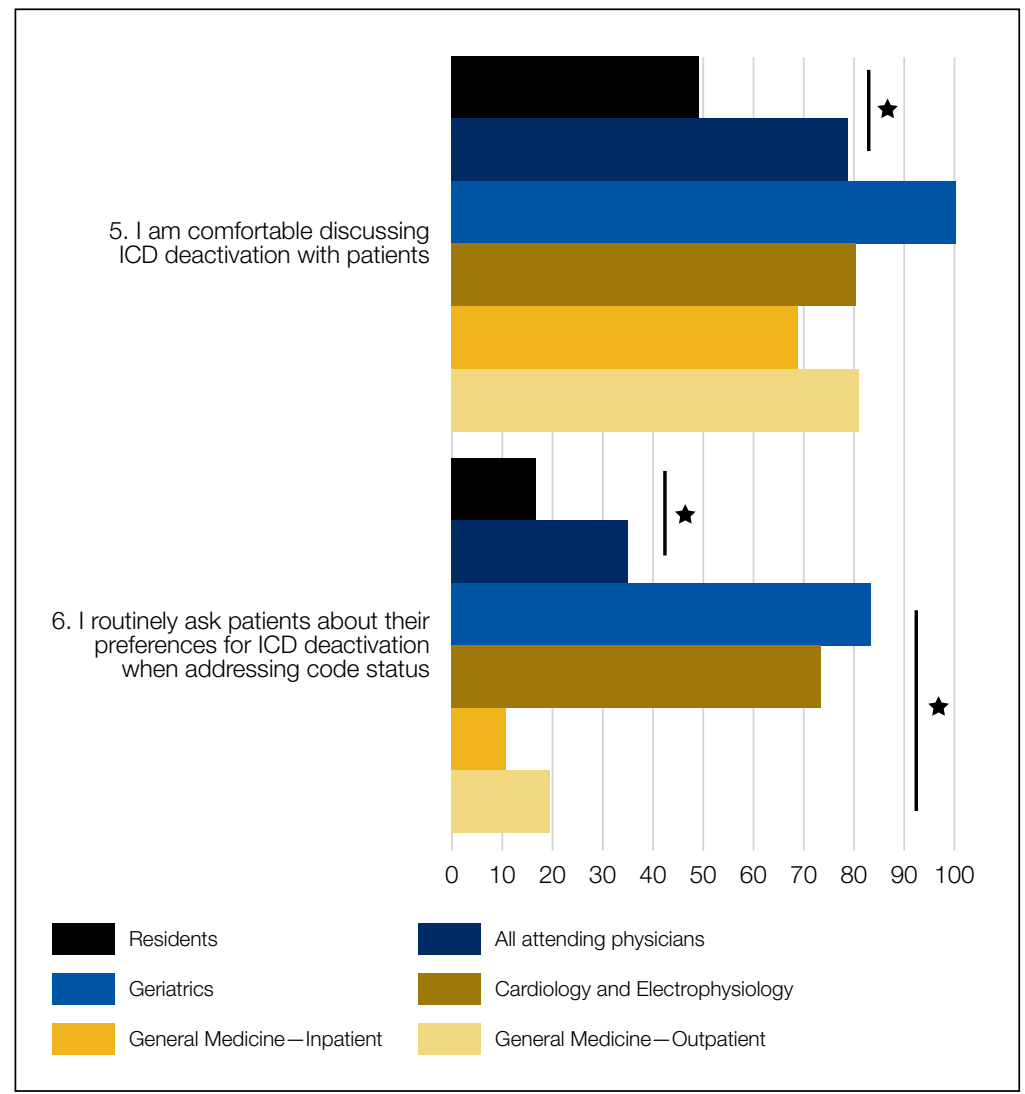

FIG. 2. Stratified responses to questions 5 and 6 . The top 2 bars represent the residents and all attending physicians. The bottom 4 bars are attending physicians stratified by specialty. Residents are less comfortable than attending physicians asking about ICD deactivation ( $P$ $<.001$; question 5). Additionally, residents ask about ICD deactivation less routinely than attending physicians $(\mathrm{P}=.018$; question 6$)$. Attending physicians of different specialties differ with respect to how routinely they ask about ICD deactivation $(P<.001)$. X-axis indicates the percentage giving an affirmative answer. Stars indicate statistically significant between-group 


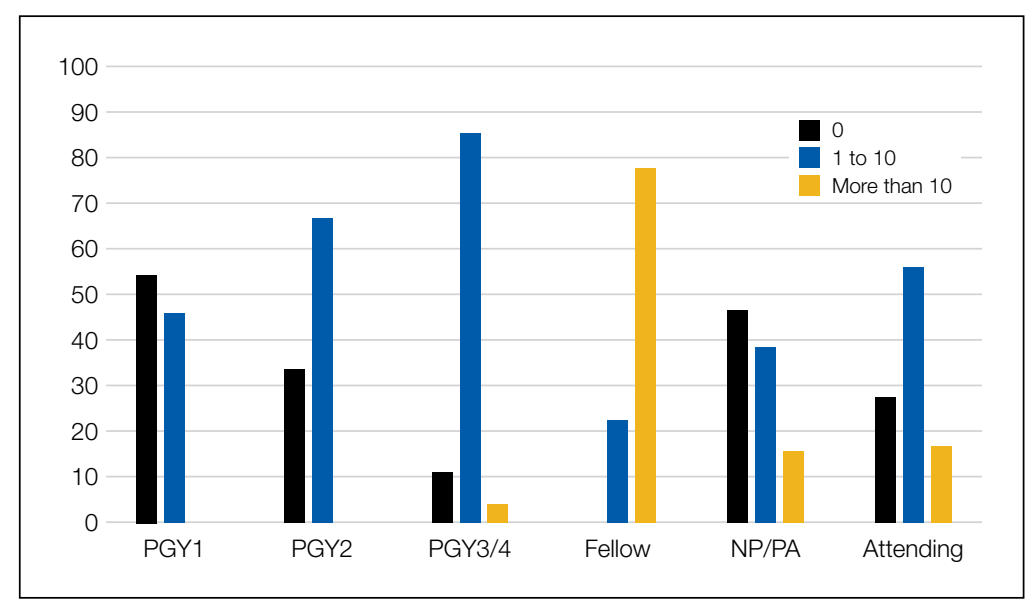

FIG. 3. The number of ICD deactivations respondents were directly involved in, stratified by training level. For conciseness, respondents who answered 1 to 5 and 5 to 10 are shown together. Although fellows appear to be the most experienced, all of them were in either cardiology or electrophysiology. The overall difference among the groups was statistically significant $(P<$ .001). Y-axis indicates the percentage in each range.

activated, and 28\% felt that non-terminally ill DNR/DNI patients should have their device deactivated.

Groups were unequal with respect to the number of ICD deactivations in which they had been directly involved (Figure $3 ; P<.001)$. Over half of interns had not been involved in any ICD deactivations as compared with only $10.7 \%$ of third- or fourth-year residents. Of the 20 geriatricians, cardiologists, and electrophysiologists, $45 \%$ had been involved in at least 5 ICD deactivations. Of note, although $77.8 \%$ of fellows reported being involved in more than 10 ICD deactivations, these 9 respondents were all in cardiology or electrophysiology.

\section{DISCUSSION}

Overall, our major findings were (1) residents, who provide much of the clinical care in a teaching hospital, are remarkably uncomfortable discussing ICD deactivation, (2) general internists and residents ask about ICD deactivation infrequently compared to geriatricians and cardiologists, and (3) about one fifth of our respondents believe ICD deactivation is automatically part of a DNR/DNI order.

Although the majority of respondents did not routinely address ICD deactivation in conjunction with code status, there was significant variability among subgroups. For example, $83.3 \%$ of geriatricians routinely discussed ICD deactivation as part of code status compared with only $4 \%$ of first-year residents and $10.5 \%$ of inpatient general internists. This finding is interesting because $90.7 \%$ of all respondents believed that discussions of code status should address preferences on ICD deactivation. This apparent discrepancy could be explained by the relatively small number of patients admitted to the hospital who have both an ICD and a request to be DNR/ DNI. Residents and inpatient general internists see a very broad spectrum of patients; ICD deactivation is frequently irrelevant in the cases these physicians manage. The subset of patients seen in consultation by cardiologists and geriatricians, by contrast, is expected to include a larger proportion of patients with ICDs. Therefore, discussing ICD deactivation will be more relevant to their daily practice. Fear of alienating patients was not a reason for our findings, as our respondents did not express concern that recommending ICD deactivation would harm the patient-clinician relationship.

There are several possible reasons that residents, particularly interns, are uncomfortable discussing ICD deactivation. A lack of exposure to ICD deactivation is probably a key contributor. Over half of interns had never been involved in any ICD deactivations. Residents and hospitalists may also feel as if they are overstepping their boundaries to discuss deactivating ICD therapies. Their feelings may not be misplaced, as one survey of ICD patients found that over $75 \%$ thought responsibility for discussing ICD deactivation, at least at the end of life, rests with cardiologists or electrophysiologists. ${ }^{6}$

The HRS guidelines call for individualized decisions regarding ICD deactivation, even if a patient is DNR/DNI. However, our respondents frequently felt a standardized approach was indicated, with $21 \%$ believing that a DNR/DNI order included ICD deactivation. Additionally, 28\% agreed that even non-terminally ill DNR/DNI patients should have their device deactivated. This is relevant because it is the role of clinicians to engage in shared decision-making with their patients. If the clinician holds the fixed belief that a DNR/ DNI order, regardless of the precise clinical scenario, should include ICD deactivation, they may pressure a patient to have their device deactivated even if it could still benefit them.

In 2009, Kelley et al published results of a survey on ICD deactivation at the end of life. ${ }^{9}$ They contacted 4,876 attending physicians in cardiology, electrophysiology, geriatrics, and general medicine, receiving 558 responses. The survey included Likert-scale questions assessing attitudes and knowledge about ICD functionality. Demographic information was also collected, including how many patients in their practice had ICDs and how often they had previously discussed ICD deactivation.

There are some interesting comparisons between Kelley et al's findings and ours, although we included trainees and the precise wording of questions was different. The specific questions used by Kelley et al to ask whether ICD shocks were painful or distressing and to ask if ICD deactivation is part of a DNR order were: "The shock from an ICD is very painful for most patients." "The shock of an ICD at the end of life is distressing to a patient and their loved ones." "A DNR order is equivalent to deactivation of an ICD."

Only $47 \%$ of general internists in the Kelley et al survey thought that ICD shocks were painful, compared with $83 \%$ of electrophysiologists. In addition, $65 \%$ of general internists and $85 \%$ of electrophysiologists viewed shocks at the 
end of life to be distressing to patients and families. By contrast, our respondents were nearly unanimous in believing shocks to be painful and distressing. This discrepancy may be due to the growing prevalence of ICDs over the past several years as well as the growing body of literature on unnecessary shocks at the end of life. In line with our study, 19\% of their respondents believed a DNR order was equivalent to ICD deactivation. ${ }^{9}$

Taken together, our findings indicate that additional education for clinicians of all levels could be helpful. Didactic lessons cannot replace experience, and it is important for residents to be exposed to discussions of ICD deactivation. However, lessons about ICD therapies and practical examples of how to broach the topic of deactivation could be beneficial, especially for interns whose responsibility includes discussions of code status. Within the context of an internal medicine residency, the fundamentals of ICD functionality could be covered during rotations on cardiology or palliative care services. Additionally, the recommendations of the HRS for device management can be covered in didactic sessions. Similar opportunities could be built into continuing medical education for practicing physicians and the training of advanced practice providers.

There are limitations to this survey, most notably the fact that it was restricted to a single academic medical center, the patient population and practices of which may not be generalizable to medical practice at large. Selection bias is also a distinct possibility given the $32 \%$ overall response rate; those who responded may feel more strongly about the survey topic. Our study subgroups may have interpreted questions differently because of their particular area of clinical practice. The small sample size also precluded an effective analysis of fellows and advanced practice practitioners due to lack of power. A major strength of this survey was the inclusion of a large number of residents upon whom the majority of inpatient contact rests. Future work could include expanding the survey to multiple medical centers, which would enhance generalizability and improve the ability to recruit sufficient fellows and advanced practice providers.

\section{CONCLUSION}

In summary, we conducted a single-center survey of residents, fellows, advanced practice providers, and attending physi- cians on their attitudes and beliefs about ICD deactivation in DNR/DNI patients. Residents are particularly uncomfortable discussing ICD deactivation with patients, which is an important finding because of their crucial role in providing patient care. Additionally, residents and hospitalists do not broach the topic of deactivation regularly, especially when compared to geriatricians and cardiologists. Despite HRS guidelines to the contrary, a fifth of our respondents believed that DNR/DNI orders include ICD deactivation. Overall, ICD deactivation in DNR/DNI patients is a topic that needs further attention in clinical education so that patients receive care that respects their individual wishes.

Disclosure: Nothing to report.

\section{References}

1. Freeman JV, Wang Y, Curtis JP, Heidenreich PA, Hlatky MA. Physician procedure volume and complications of cardioverter-defibrillator implantation. Circulation. 2012;125(1):57-64. doi:10.1161/CIRCULATIONAHA.111.046995.

2. Kremers MS, Hammill SC, Berul CI, et al. The National ICD Registry Report: Version 2.1 including leads and pediatrics for years 2010 and 2011. Hear Rhythm. 2013;10(4):e59-e65. doi:10.1016/j.hrthm.2013.01.035.

3. Goldstein NE, Mehta D, Siddiqui S, et al. "That's like an act of suicide" patients' attitudes toward deactivation of implantable defibrillators. J Gen Intern Med. 2008;23 Suppl 1:7-12.

4. Goldstein NE, Lampert R, Bradley E, Lynn J, Krumholz HM. Management of implantable cardioverter defibrillators in end-of-life care. Ann Intern Med. 2004;141(11):835-838. http://annals.org/article.aspx?articleid=717985\& issueno=11. Accessed October 23, 2013.

5. Sherazi S, Daubert JP, Block RC, et al. Physicians' preferences and attitudes about end-of-life care in patients with an implantable cardioverter-defibrillator. Mayo Clin Proc. 2008;83(10):1139-1141. doi:10.4065/83.10.1139.

6. Kirkpatrick JN, Gottlieb M, Sehgal P, Patel R, Verdino RJ. Deactivation of implantable cardioverter defibrillators in terminal illness and end of life care. Am J Cardiol. 2012;109(1):91-94. doi:10.1016/j.amjcard.2011.08.011.

7. Marinskis G, van Erven L. Deactivation of implanted cardioverter-defibrillators at the end of life: results of the EHRA survey. Europace. 2010;12(8):1176-1177. doi:10.1093/europace/euq272.

8. Mueller PS, Jenkins SM, Bramstedt KA, Hayes DL. Deactivating implanted cardiac devices in terminally ill patients: practices and attitudes. Pacing Clin Electrophysiol. 2008;31(5):560-568. doi:10.1111/j.1540-8159.2008.01041.x.

9. Kelley AS, Reid MC, Miller DH, Fins JJ, Lachs MS. Implantable cardioverter-defibrillator deactivation at the end of life: a physician survey. Am Heart J. 2009;157(4):702-8.e1. doi:10.1016/j.ahj.2008.12.011.

10. Lampert R, Hayes DL, Annas GJ, et al. HRS Expert Consensus Statement on the Management of Cardiovascular Implantable Electronic Devices (CIEDs) in patients nearing end of life or requesting withdrawal of therapy. Hear Rhythm. 2010;7(7):1008-1026. doi:10.1016/j.hrthm.2010.04.033.

11. Kelley AS, Mehta SS, Reid MC. Management of patients with ICDs at the end of life (EOL): a qualitative study. Am J Hosp Palliat Care. 2008;25(6):440-446. doi:10.1177/1049909108320885. 